\title{
Logro de competencias en el proceso de aprendizaje durante tiempos del COVID-19
}

\section{Achievement of competencies in the learning process during times of COVID-19}

\author{
Teresa Bautista Facho \\ Ministerio de Educación del Perú (MINEDU), Perú \\ ORCID: https://orcid.org/0000-0002-2213-6967 \\ Héctor Raúl Santa María Relaiza \\ Universidad César Vallejo, Perú. \\ ORCID: https://orcid.org/0000-0002-4546-3995 \\ Ulises Córdova García \\ Universidad César Vallejo, Perú. \\ ORCID: https://orcid.org/0000-0002-0931-7835
}

Received 10-12-20 Revised 11-25-20

Accepted 02-13-21 On line 03-19-21

*Correspondence

Email: bautistafacho@gmail.com
Cite as: 


\section{Resumen}

Este artículo presenta la investigación realizada sobre el Logro de competencias durante el proceso de aprendizaje en los tiempos del COVID-19 en la Educación Básica peruana, la cual ha pasado de una modalidad presencial a una de distancia. Para la variable Logro de competencias se han considerado cuatro dimensiones: la cognitiva, la comunicativa, la social y la ética. Se desarrolló dentro del enfoque cuantitativo; su nivel fue descriptivo explicativo, de diseño no experimental, transaccional exploratorio, aplicándose una encuesta a 212 docentes de una red educativa de Lima Metropolitana. El objetivo fue analizar el factor predominante que limita el logro de las competencias priorizadas. Aplicando la regresión logística, desde la perspectiva de los docentes se logró determinar que la retroalimentación $(0,589)$ y la evaluación del portafolio $(0,694)$ son factores que limitan el logro de las competencias, pues muestran valores de significancia mayores que la teórica $(\alpha=0,05)$, mientras que el rol de la familia $(0,00)$ no limita este logro. El docente, siendo mediador del aprendizaje, debe fomentar el desarrollo de competencias. Factores como la retroalimentación y la evaluación del portafolio de evidencias deben tener significancia en sus estudiantes; pero en esta investigación son más bien factores que están limitando el logro de competencias.

Palabras clave: logro de competencia; aprendizaje; retroalimentación; evaluación de portafolio; rol de la familia.

\section{Summary}

This paper presents a research carried out on the achievement of competencies during the learning process in times of COVID-19 in Peruvian Basic Education, which has gone from a face-to-face modality to another of distance learning. For the variable achievement of competencies, four dimensions have been considered: cognitive, communicative, social and ethical. It was developed within the quantitative approach, its level was descriptive explanatory, of non-experimental design, transactional exploratory, applying a survey to 212 teachers of an educational network in Metropolitan Lima. The objective was to analyze the predominant factor that hinders the achievement of the prioritized competencies. Applying the logistic regression, from the teachers' perspective, it was possible to determine that the feedback (0.589) and the portfolio evaluation (0.694) are factors that hinder the achievement of competencies since they show significance values greater than the theoretical $(\alpha=0.05)$, while the role of the family $(0.00)$ does not hinder this achievement. The teacher, being a learning mediator, must promote the development of competencies. Factors such as feedback and evaluation of the evidence portfolio should have significance in his/her students; but in this research they are rather factors that are limiting the achievement of competencies.

Keywords: achievement of competence; learning; feedback; portfolio evaluation; role of the family.

\section{Introducción}

Todos los países del mundo han sido afectados por una pandemia, el COVID-19, causada por un coronavirus, que afecta el desarrollo económico, social y cultural en cada país. Dentro del aspecto social, preocupa la educación; muchos niños y adolescentes han pasado de una educación presencial a una educación a distancia. Es necesario precisar que el Currículo Nacional demanda un 
perfil de egresado compuesto por 29 competencias, de las cuales se han priorizado las de ciudadanía, comunicación, ciencia y tecnología, matemática, educación física, bajo la estrategia "Aprendo en Casa" a través de la TV, radio e internet.

En medio de esta emergencia, el reto de las comunidades educativas es utilizar para el logro de competencias los medios tecnológicos y las distintas plataformas. Los docentes son los que realizan el andamiaje explorando los saberes previos de los estudiantes y conectándolos al nuevo saber, llevándolos de una zona de confort hacia la zona de desarrollo próximo (Rodríguez, 1999).

El docente, entonces, es el mediador, el que acompaña en el desarrollo de las competencias de sus estudiantes. Cabe precisar que las competencias son formas de actuar de manera integral de la persona para ejercer una serie de capacidades básicas hasta llegar a capacidades superiores con la finalidad de resolver problemas en la sociedad, desarrollando y aplicando de manera articulada el saber ser, saber convivir, saber hacer y saber conocer, en el rol que nos toca como parte de una sociedad (Tobón, 2015).

En este contexto cabe plantearnos la pregunta: “¿Cuál es el factor predominante que limita el logro de las competencias priorizadas en la educación a distancia de la Educación Básica a causa de la pandemia del COVID-19?". Para comprender la magnitud del problema nos hemos propuesto como objetivo analizar el factor predominante que limita el logro de las competencias priorizadas en la educación a distancia de la Educación Básica Regular a causa de la pandemia del COVID-19, cuyos resultados servirán de línea base para realizar mejoras en el logro de aprendizajes.

A través de la estrategia "Aprendo en Casa", aproximadamente el $50 \%$ de estudiantes siguen las actividades desde la televisión y el otro 50\% desde la web, según informes de directivos en el Sistema de Gestión de la Calidad del Servicio Educativo (SIMON) (Minedu, 2015). Surge la necesidad, en ese contexto, de hacer un análisis respecto de si existe un factor predominante que limita el logro de las competencias priorizadas en la educación a distancia de la Educación Básica a causa de la pandemia del COVID-19. Este factor puede ser la retroalimentación realizada por los docentes, la evaluación del portafolio realizado por el estudiante o el rol que juegan las familias en este contexto. La finalidad es realizar aportes para la mejora de la estrategia y superar las debilidades encontradas.

Existen factores que influyen en la calidad de los aprendizajes, es decir, en el logro de competencias, entre ellos tenemos la baja calidad docente con prácticas que no responden a las demandas; en consecuencia, el logro de competencias es mínimo o simplemente no se logra (Meléndez 2011) en estudiantes de secundaria de países de América Latina; por ejemplo, no se están logrando competencias científicas (Zúñiga, Leiton \& Naranjo 2014) o se percibe debilidades en el logro de estas (Silva, 2016). Cabe señalar que a mayor desempeño docente, se tiene un mayor logro de competencias en los estudiantes (Oscco, 2015). Dentro del enfoque del desarrollo de competencias, es necesario que el docente realice una evaluación formativa promoviendo el uso de diversas estrategias didácticas (Flores, 2017) y debe implementar un proceso evaluativo que realmente mida el logro de competencias desde dos enfoques: el conductista y el constructivista (Pantoja, 2018). La articulación entre cada elemento de la evaluación, como indicadores, instrumentos, desempeños con las evidencias recogidas, es de suma importancia (Huerta, 2018); así se podría conocer y analizar si se ha empezado a desarrollar la competencia. Por otro lado, es importante recalcar la alta expectativa del docente hacia el estudiante: si en los estudiantes talentosos se profundiza en la competencia Afán de logro de acuerdo a sus habilidades, conseguirán gran notoriedad en donde laboren. Recordemos que todas las personas tienen talentos, y en esta tarea de estimularlos se complementan el trabajo del docente con el de la familia, lo que favorece el proceso de enseñanza-aprendizaje (García, Ayestarán; López \& Tovar, 2019).

Debemos señalar que los pilares de la educación deben estar inmersos en cada miembro de la comunidad educativa para lograr una buena gestión y brindar educación de calidad, en otros términos, logros de competencias para la vida (García, Juárez \& Salgado, 2018). En estos tiempos 
de la pandemia del COVID-19, la familia participa en los procesos educativos de la escuela supervisando el aprendizaje en casa (Miranda \& Castillo 2018); los padres tienen un rol muy importante, ellos son los que hacen respetar las normas en casa, tienen una visión del uso moderado de las TIC; y si bien es cierto que los hijos son nativos digitales, son los padres quienes no pierden de vista la educación de sus hijos y actuando muchas veces como facilitadores, los ayudan, pues ven de cerca la motivación en ellos al realizar sus producciones o actuaciones en el marco de la estrategia Aprendo en Casa, modalidad de educación a distancia (Sánchez, De Andrés \& Paredes, 2018).

Es muy importante dar una mirada retrospectiva en la historia, en lo que se refiere a generar aprendizaje, de tal forma que este se encuentre articulado con las necesidades e intereses de los estudiantes. En este sentido, los filósofos griegos ya abordaban los temas esenciales a partir de problemas contextualizados (Tobón, 2015).

La línea histórica de competencias diríamos que surge desde las civilizaciones antiguas. Hay términos muy parecidos al de competencia, por ejemplo en el Código de Hammurabi (17921750 a.C.), de la civilización de Mesopotamia, está la palabra ikanótis 'habilidad de conseguir algo'. En el siglo XVI tenemos el término competencia asociado a la capacidad general para realizar una actividad o resolver un problema en un curso. En la década de 1960 dicha palabra tiene uso en la terminología lingüística (como la capacidad de entender y hablar una lengua) y en el aprendizaje. Década de 1970: se da en el campo de la gestión del talento humano en diversas organizaciones y empieza a aplicarse en el campo educativo. Década de 1980: en referencia a que los trabajadores mejoran sus desempeños laborales a través de proyectos. Década de 1990: en los distintos países empieza la generación de modelos curriculares que responden a necesidades identificadas. Década del 2000: los diferentes estados en sus políticas educativas incorporan el concepto de competencias desde un enfoque holístico (Rychen y Salganik, 2003) (Tobón, 2015).

El término competencia tiene una larga historia y hoy en día plantea que el aprendizaje sea autónomo, significativo, que la persona logre una idoneidad y ética para que sea capaz de afrontar retos; se entiende que el ser humano continúa aprendiendo y realizando tareas con mayor complejidad a lo largo de su vida (Ormrod, Sanz, Soria \& Carnicero, 2005).

Las actuales demandas implican que el docente juegue otro rol, busque otras estrategias, otras formas de enseñanza, y que se convierta en un mediador del aprendizaje. Así, la teoría de Piaget contribuye a potenciar el desarrollo de métodos de enseñanza que estimularán el aprendizaje activo, en el cual el sujeto de forma activa construye su propio aprendizaje (Rodríguez, 1999), complementando con la actividad del profesor, quien propone entornos de aprendizaje y actividades adaptadas al nivel de desarrollo de sus estudiantes. Los futuros docentes deben ser formados, fortalecidos, en el uso pedagógico de las TIC; no basta con saber sobre ellas sino implica utilizarlas porque son un soporte para el aprendizaje, sobre todo para esta modalidad, la educación a distancia, la cual supone un manejo amplio de las TIC (Saldarriaga, Bravo \& Loor, 2016) (Flores \& Roig, 2019).

Todos los docentes de la Educación Básica tienen en el Currículo Nacional la columna vertebral que orienta su labor; en este documento se define que las competencias movilizan un conjunto de capacidades para lograr un propósito actuando pertinentemente, con principios, valores, con sentido ético (Minedu, 2016) (Tobón, Prieto \& Fraile, 2010).

Desde la perspectiva del enfoque socioformativo, las competencias son actuaciones integrales ante retos, desafíos y problemas del contexto, que exigen un sentido ético, integrando cuatro saberes: el saber ser, el saber hacer, el saber convivir y el saber conocer bajo el criterio de mejorar continuamente. Es necesario que las comunidades educativas reflexionen al respecto, que se tenga claro qué es necesario para desarrollar competencias en nuestros estudiantes, partiendo desde una misma autoevaluación del actuar del docente (¿qué tipos de prácticas pedagógicas está desarrollando?, ¿las contextualiza?, ¿utiliza las herramientas necesarias?) (Tobón et al. 2010). 
En el Perú, las competencias ya están determinadas en el Currículo Nacional; sin embargo, los docentes deben interiorizarlas y ponerlas en práctica, lo que motiva las preguntas: "¿Qué implicancias tiene cada una de ellas?”, “¿Cuál es el propósito de aprendizaje?”, ¿Hacia dónde voy?”, “¿Cómo las voy a evaluar?”, “¿Qué evidencias debo recoger que me permitan afirmar que se está logrando desarrollar las competencias?”, ¿Lograron llegar a mi propósito?”, “¿Qué aspectos han quedado débiles para retroalimentar?"

Dentro del desarrollo de competencias, se deben tener muy claros los criterios de evaluación, ya que estos son las pautas fundamentales que se han de tomar en cuenta para valorar la competencia, el estándar y/o el desempeño (Tobón et al., 2010).

Valorar una competencia implica saber qué desempeños se espera de los estudiantes para poder contrastar con los estándares. Sin embargo, existe una confusión por parte de docentes entre los conceptos de evaluación y calificación; falta delimitar los criterios y la inmersión de los estudiantes en el proceso educativo (Hortigüela, Pérez y González, 2019).

Dentro de la evaluación progresiva de la competencia existen pruebas concretas y tangibles de que se está logrando una competencia; estas son las evidencias que se evalúan con base en los criterios, y es necesario valorarlas en forma integral y no de manera aislada (Tobón et al. 2010), recordando que existe articulación entre cada elemento de la evaluación: indicadores, instrumentos, desempeños con las evidencias recogidas (Huerta, 2018).

Estas evidencias se van organizando en el portafolio de evidencias, sintetizando de manera crítica cada una de ellas (Tobón, 2015; Arceo \& Bravo, 2014). Mediante el portafolio, los docentes y estudiantes pueden monitorear el proceso de construcción y reconstrucción de la competencia; esto, además, constituye un proceso interactivo entre ellos y hace que la evaluación sea realmente un proceso formativo con retroalimentación oportuna, retroalimentación formativa que permita interacciones donde los estudiantes producen o actúan, identificando fortalezas y debilidades y los docentes en un dialogo reflexivo orientan hacia el desarrollo de la autonomía (Anijovich, \& Cappelletti, 2017), en entornos virtuales de aprendizaje, como es el de la demanda de la educación a distancia, en la cual la retroalimentación se ajusta a las necesidades de los estudiantes cuando aprenden conocimientos conceptuales (Máñez, 2019).

Es muy importante también abordar la estrategia Aprendo en Casa como una posibilidad de que estudiantes de la Educación Básica regular sigan desarrollando desde sus hogares las competencias priorizadas, respetando los protocolos de aislamiento social, proceso en que los padres de familia juegan un rol muy importante, ya que sin ser docentes o pedagogos deben ser mediadores del aprendizaje de sus hijos y acompañarlos en estos nuevos retos que la misma necesidad lo requiere. Así mismo, debe resaltarse que la familia tiene entre sus roles la tarea de trasmitir a niños, adolescentes y jóvenes valores éticos, sociales y culturales, para una convivencia adecuada, aspecto importante en el aprendizaje de sus hijos y lo que implica el logro de competencias (López \& Guiamaro, 2017). La familia cumple su función educadora participando en los procesos educativos de la escuela, supervisando el aprendizaje en casa (Miranda \& Castillo 2018).

\section{Método}

El enfoque de la investigación sobre el logro de competencias durante el proceso de aprendizaje en los tiempos del COVID-19 fue cuantitativo; se pretendió determinar si existe un factor predominante que limitó el logro de las competencias priorizadas en la educación a distancia de la Educación Básica a causa de la pandemia originada por el coronavirus. Fue un estudio de tipo exploratorio al tratarse de una situación nueva y descriptiva, porque se pretendió medir el logro de competencias de la estrategia Aprendo en Casa. La muestra estuvo constituida por 212 docentes de instituciones públicas en condición de nombrados y contratados que están laborando con estudiantes 
de la Educación Básica; el muestreo fue probabilístico, ya que cualquier elemento de la población tiene la misma probabilidad de salir (Hernández et al., 2008). En esta investigación se trabajó con la Red Educativa Institucional N. ${ }^{\circ}$ 10, correspondiente a instituciones del distrito de San Martín de Porres. Se utilizó un cuestionario con escala de Likert conformado por 12 preguntas que abordan las 4 dimensiones de la variable Logro de competencias: cognitiva, comunicativa, social y ética, cada una de ellas con tres indicadores y factores como la retroalimentación, la evaluación del portafolio y la intervención de la familia, lo que fue validado en pertinencia, relevancia y claridad por el juicio de tres expertos. Así mismo, se utilizó el procedimiento Coeficiente alfa de Cronbach, que arroja una fiabilidad de 0,78 con 37 casos válidos que implican el 100\%.

\section{Resultados}

\section{Tabla 1.}

Factores que limitan el logro de competencias durante el proceso de aprendizaje en los tiempos del COVID-19

\begin{tabular}{lll}
\hline Nivel & Frecuencia & Porcentaje \\
\hline Totalmente en desacuerdo & 1 & $0,47 \%$ \\
En desacuerdo & 10 & $4,72 \%$ \\
De acuerdo & 184 & $86,79 \%$ \\
Totalmente de acuerdo & 17 & $8,02 \%$ \\
Total & $\mathbf{2 1 2}$ & $\mathbf{1 0 0 \%}$ \\
\hline
\end{tabular}

Fuente: Cuestionarios aplicados a los docentes.

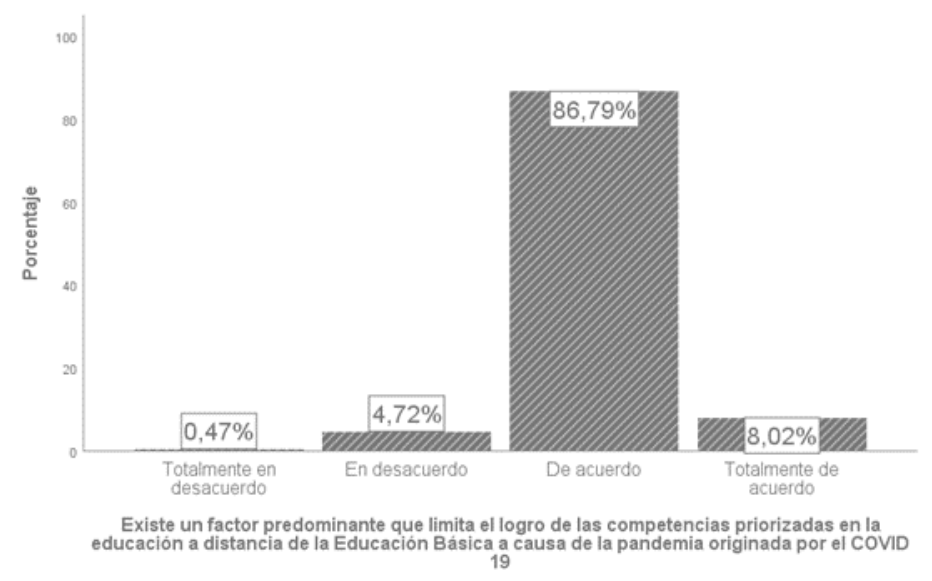

Figura 1. Factores que limitan el logro de competencias durante el proceso de aprendizaje en los tiempos del COVID-19. 
De la Tabla y Figura 1, se observa que el $86,79 \%$ de los docentes encuestados están de acuerdo en que existen factores que limitan el logro de las competencias, mientras que un $0,47 \%$ están en totalmente en desacuerdo.

\section{Tabla 2.}

El factor predominante que limita el logro de las competencias priorizadas en la estrategia Aprendo en Casa es la retroalimentación realizada por los docentes.

\begin{tabular}{lcc}
\hline \multicolumn{1}{c}{ Nivel } & Frecuencia & Porcentaje \\
\hline Totalmente en desacuerdo & 2 & $0,94 \%$ \\
En desacuerdo & 12 & $5,66 \%$ \\
De acuerdo & 175 & $82,55 \%$ \\
Totalmente de acuerdo & 23 & $10,85 \%$ \\
Total & $\mathbf{2 1 2}$ & $\mathbf{1 0 0 \%}$ \\
\hline
\end{tabular}

Fuente: Cuestionarios aplicados a los docentes.

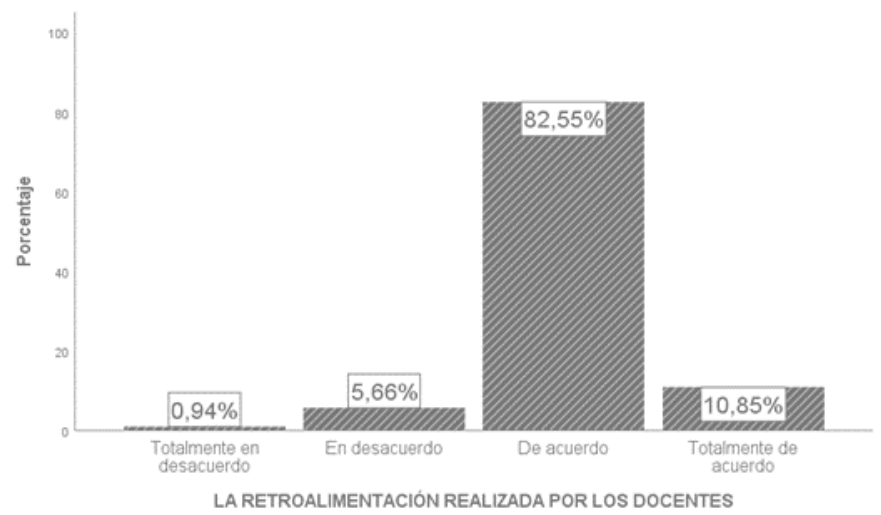

Figura 2. El factor predominante que limita el logro de las competencias priorizadas en la estrategia Aprendo en Casa es la retroalimentación realizada por los docentes

De la Tabla y Figura 2, se observa que el $82,55 \%$ de los docentes encuestados están de acuerdo en que el factor predominante que limita el logro de las competencias priorizadas en la estrategia Aprendo en Casa es la retroalimentación realizada por los docentes, mientras que el $0,94 \%$ están totalmente en desacuerdo. 


\section{Tabla 3.}

El factor predominante que limita el logro de las competencias priorizadas en la estrategia Aprendo en Casa es la evaluación del portafolio realizado por los estudiantes

\begin{tabular}{lcc}
\hline \multicolumn{1}{c}{ Nivel } & Frecuencia & Porcentaje \\
\hline Totalmente en desacuerdo & 1 & $0,47 \%$ \\
En desacuerdo & 10 & $4,72 \%$ \\
De acuerdo & 136 & $64,15 \%$ \\
Totalmente de acuerdo & 65 & $30,66 \%$ \\
Total & $\mathbf{2 1 2}$ & $\mathbf{1 0 0 \%}$ \\
\hline
\end{tabular}

Fuente: Cuestionarios aplicados a los docentes

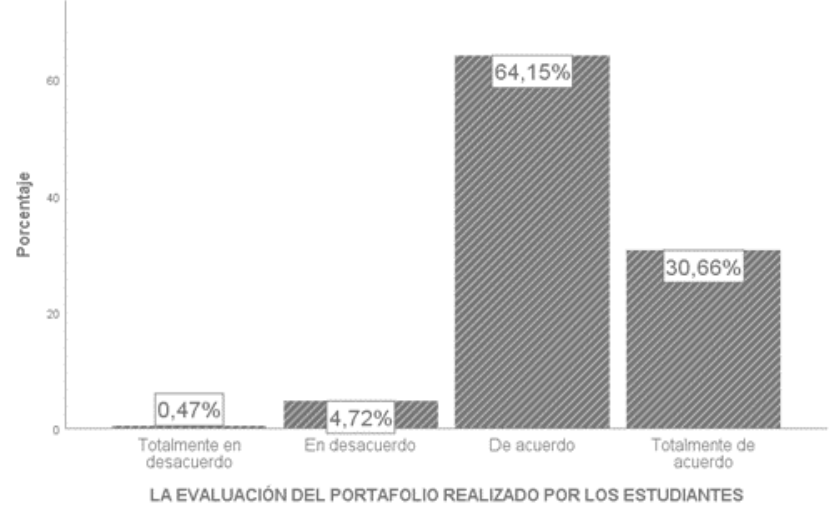

Figura 3. El factor predominante que limita el logro de las competencias priorizadas en la estrategia Aprendo en Casa es la evaluación del portafolio realizado por los estudiantes.

De la Tabla y Figura 3, se observa que el $64,15 \%$ de los docentes encuestados están de acuerdo en que el factor predominante que limita el logro de las competencias priorizadas en la estrategia Aprendo en Casa es la evaluación del portafolio realizado por los estudiantes mientras que el $0,47 \%$ están totalmente en desacuerdo.

Tabla 4.

El factor predominante que limita el logro de las competencias priorizadas en la estrategia Aprendo en Casa es el rol de las familias.

\begin{tabular}{lcc}
\hline \multicolumn{1}{c}{ Nivel } & Frecuencia & Porcentaje \\
\hline Totalmente en desacuerdo & 2 & $0,94 \%$ \\
En desacuerdo & 56 & $26,42 \%$ \\
De acuerdo & 146 & $68,87 \%$ \\
Totalmente de acuerdo & 8 & $3,77 \%$ \\
Total & $\mathbf{2 1 2}$ & $\mathbf{1 0 0 \%}$ \\
\hline
\end{tabular}

Fuente: Cuestionarios aplicados a los docentes. 


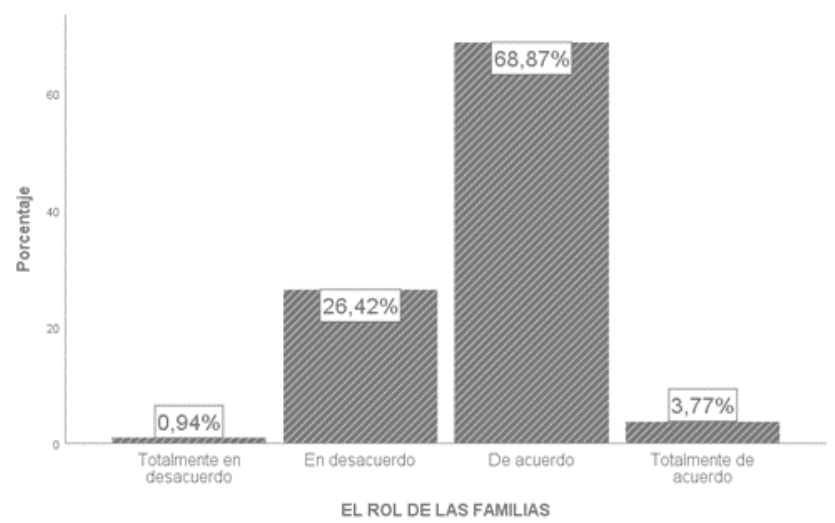

Figura 4. El factor predominante que limita el logro de las competencias priorizadas en la estrategia Aprendo en Casa es el rol de las familias.

De la Tabla y Figura 4, se observa que el $68,87 \%$ de los docentes encuestados están de acuerdo en que el factor predominante que limita el logro de las competencias priorizadas en la estrategia Aprendo en Casa es el rol de las familias, mientras que el 0,94\% están totalmente en desacuerdo.

\section{Función de Prueba}

Se realizó por medio de la regresión logística, ya que el factor predominante (o factor que aporta más) es el factor con el coeficiente de regresión logística de mayor valor sin tomar en cuenta el signo (es decir, en valor absoluto).

El coeficiente de regresión logística muestra el peso que tiene cada factor sobre la variable de estudio, lo cual significa que cuando el coeficiente es más grande, el peso sobre la variable también es grande; caso contrario, si el coeficiente es pequeño, el peso sobre la variable también es pequeño o, dicho de otra manera, un coeficiente alto incrementa la probabilidad de que un encuestado (o evaluado) tenga un nivel bajo en la variable respecto a otro encuestado (o evaluado) que tenga un nivel alto en la variable.

Por otro lado, el valor de odds ratio $\langle\operatorname{Exp}(B)>$ muestra si el factor es protectora o de riesgo. Protectora si $\operatorname{Exp}(B)$ es menor que 1, riesgo si $\operatorname{Exp}(B)$ es mayor que 1, siempre y cuando cumpla con que la unidad no esté incluida en el intervalo de confianza del $\operatorname{Exp}(\mathrm{B})$.

\section{i. Regla de decisión}

Rechazar $\mathrm{H}_{0}$ cuando la significación observada " $p$ ” de los coeficientes del modelo logístico es menor que $\alpha$.

No rechazar $\mathrm{H}_{0}$ cuando la significación observada " $p$ " de los coeficientes del modelo logístico es mayor que $\alpha$.

\section{ii. Cálculos}


Tabla 5.

Coeficientes de la regresión logística de los factores que limitan el logro de las competencias a distancia de la Educación Básica a causa de la pandemia del COVID-19.

\begin{tabular}{ccccccc}
\hline Factor & B & $\begin{array}{c}\text { Error } \\
\text { estándar }\end{array}$ & Sig. & Exp(B) & \multicolumn{2}{c}{$\mathbf{9 5 \% \text { C.I. para EXP(B) }}$} \\
\cline { 5 - 7 } & & Inferior & Superior \\
\hline $\begin{array}{c}\text { La retroalimentación realizada } \\
\text { por los docentes }\end{array}$ & 0,436 & 0,805 & 0,589 & 1,546 & 0,319 & 7,497 \\
$\begin{array}{c}\text { La evaluación del portafolio } \\
\text { realizado por los estudiantes } \\
\text { El rol de las familias }\end{array}$ & 0,239 & 0,608 & 0,694 & 1,270 & 0,386 & 4,182 \\
\hline
\end{tabular}

Fuente: Base de datos

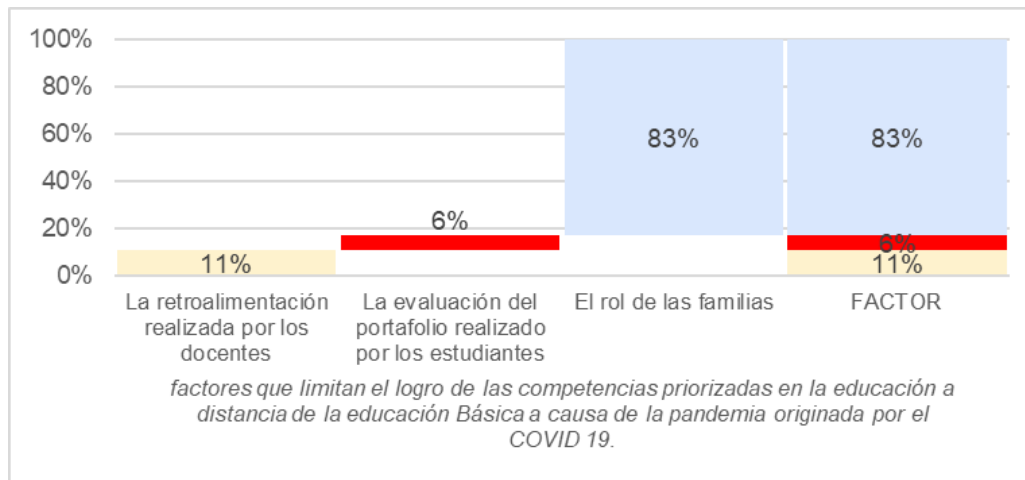

Figura 5. Pesos de los factores que limitan el logro de las competencias durante el proceso de aprendizaje en los tiempos del COVID-19.

Analizando la Tabla 5 nos damos cuenta de que el factor Rol de las familias con un valor de significancia de 0,00 menor que el valor de significancia no limita el logro de estas competencias; al contrario, es un factor que facilita el logro del desarrollo de competencias. Por tanto, podemos afirmar que el factor de mayor peso es el rol de las familias.

\section{Discusión}

Como uno de los valores de significación observada $(0,00)$ es menor que el valor de significación teórica $\alpha=0,05$, se rechaza la hipótesis nula. Por consiguiente, existe un factor predominante que no limita el logro de las competencias priorizadas en la educación a distancia de la Educación Básica a causa de la pandemia originada por el coronavirus. El logro de competencias implica que el docente, siendo mediador entre el que aprende y el conocimiento, logra que la retroalimentación que brinda signifique en los estudiantes el haber articulado sus saberes: el ser, el convivir, el hacer y el saber conocer. Según afirman Tobón et al. (2010), al contrastar con el objetivo de esta investigación, que es analizar el factor predominante que limita el logro de las competencias priorizadas en la educación a distancia de la Educación Básica Regular a causa de la pandemia del COVID 19, nos encontramos que desde la perspectiva de los mismos docentes la retroalimentación está limitando el logro de las competencias; entonces, es probable que por la 
modalidad en que se están desarrollando las sesiones de aprendizaje, la necesidad de conectividad y el uso de tecnología, no se logre la articulación de los saberes.

En términos generales, de los tres factores abordados en esta investigación, dos de ellos la retroalimentación, con una significancia observada de 0,589 mayor que el valor de significancia teórica $(0,05)$, y la evaluación del portafolio con un valor de significancia de 0,694 mayor que el valor de significancia teórica $(0,05)$ - son factores que limitan el logro de las competencias priorizadas en la estrategia Aprendo en Casa. Así mismo, respecto de la evaluación del portafolio realizado por los estudiantes — que en esta investigación es un factor limitante para el logro de competencias-, esto implica que los docentes tal vez no tienen esa práctica que permita contrastar los desempeños de sus estudiantes con las competencias, con los estándares o con los criterios que él mismo ha establecido enmarcados en lo que implica una evaluación formativa para el logro de competencias, difiriendo de Anijovich, R. \& Cappelletti, G. (2017).

El factor Rol de las familias con un valor de significancia de 0,00, menor que el valor de significancia, no limita el logro de estas competencias; al contrario, es un factor que permite el logro del desarrollo de competencias. Es importante volver a resaltar que la familia ha logrado, según esta investigación, jugar ese rol orientador en los procesos de aprendizaje en esta modalidad a distancia; y es un factor que no limita el logro de competencias, supervisando, manteniendo valores éticos, sociales y culturales, para una convivencia adecuada, aspecto importante en el aprendizaje de sus hijos y que implica el logro de competencias, coincidiendo con López \& Guiamaro (2017).

\section{Conclusiones}

El objetivo de esta investigación fue analizar el factor predominante que limita el logro de las competencias priorizadas en la educación a distancia de la Educación Básica Regular a causa de la pandemia del COVID-19. Uno de los factores predominantes que limita el logro de las competencias priorizadas en la educación a distancia de la Educación Básica a causa de la pandemia del COVID-19 es la evaluación del portafolio con evidencias, realizada por los docentes con una significancia observada de 0,694 , mayor que el valor de significancia teórica $(0,05)$. Otro factor predominante que limita el logro de las competencias priorizadas en la educación a distancia de la Educación Básica a causa de la pandemia del COVID-19 es la retroalimentación realizada por los docentes, con una significancia observada de 0,589, mayor que el valor de significancia teórica $(0,05)$. Encontramos que desde la perspectiva de los mismos docentes, la retroalimentación está limitando el logro de las competencias; es probable que por la modalidad en que se están desarrollando las sesiones de aprendizaje, la necesidad de conectividad y el uso de tecnología, no se esté llegando a lograr la articulación de los saberes. El factor que no limita el logro de competencias priorizadas en la educación a distancia de la Educación Básica a causa de la pandemia originada por el COVID-19, es rol de las familias, con un valor de significancia de 0,00, menor que el valor de significancia.

Se recomienda al ministerio de Educación fortalecer las competencias de los docentes en lo que se refiere a retroalimentación y evaluación del portafolio de evidencias dentro de la evaluación formativa y, posteriormente, realizar otras investigaciones relacionadas con esta, cuya única finalidad es el logro de las competencias en el proceso de aprendizaje de nuestros estudiantes de la Educación Básica Regular.

\section{Referencias}

Anijovich, R., \& Cappelletti, G. (2017). La evaluación como oportunidad. Buenos Aires: Paidós.

Arceo, F. D. B., \& Bravo, R. B. (2014). Diseño y validación de una propuesta de evaluación auténtica de competencias en un programa de formación de docentes de educación básica en México. 
Perspectiva Educacional, Formación de Profesores, 53(1), 36-56.

https://www.redalyc.org/articulo.oa?id=333329700004

Flores, C. y Roig, R. (2019). Factores personales que inciden en la autovaloración de futuros maestros sobre la dimensión pedagógica del uso de TIC. Revista iberoamericana de educación superior, 10(27), 151-171.

http://www.scielo.org.mx/scielo.php?pid=S2007-28722019000100151\&script=sci_arttext

García, F. J., Juárez, S. C., \& Salgado, L. (2018). Gestión escolar y calidad educativa. Revista Cubana de Educación Superior, 37(2), 206-216. http://scielo.sld.cu/scielo.php?script=sci_arttext\&pid=S0257-43142018000200016

García, M. L., Ayestarán C, R., López, J. E., \& Tovar, M. (2019). Educar y formar al alumno talentoso: El afán de logro como competencia curricular. Comunicar, 60(2019-3), 1-12. DOI https://doi.org/10.3916/C60-2019-02

Hernández, R, Fernández C, Baptista, P. (2008). Metodología de la investigación. México: McGrawHill.

Hortigüela, D., Pérez, Ángel \& González, G. (2019). Pero... ¿A qué nos Referimos Realmente con la Evaluación Formativa y Compartida?: Confusiones Habituales y Reflexiones Prácticas. Revista Iberoamericana De Evaluación Educativa, $12(1)$. https://doi.org/10.15366/riee2019.12.1.001

Huerta, M. (2018). Evaluación basada en evidencias, un nuevo enfoque de evaluación por competencias. Revista de Investigaciones de la Universidad Le Cordon Bleu, 5(1), 159-171. https://doi.org/10.36955/RIULCB.2018v5n1.0011

López, G., \& Guiamaro, Y. (2017). El rol de la familia en los procesos de educación y desarrollo humano de los niños y niñas. Ixaya. Revista Universitaria de Desarrollo Social, (10), 3155.

https://www.uam.mx/cdi/pdf/redes/xi_chw/cendif_rol.pdf

Máñez, I. (2019). ¿Influye la retroalimentación correctiva en el uso de la retroalimentación elaborada en un entorno digital? Educational Psychology, 26(1), 57-65.

https://journals.copmadrid.org/psed/art/psed2019a14\#: :text=Parece\%20que\%20proporcion ar\%20retroalimentaci\%C3\%B3n\%20que,et\%20al.\%2C\%202015).

Meléndez Olivari, G. M. (2011). La gestión del acompañamiento pedagógico: el caso del programa estratégico logros de aprendizaje al finalizar el III ciclo de educación básica regular (PELA) en la región Callao-UGEL Ventanilla.

Minedu (2015). Sistema de monitoreo y evaluación de la calidad del servicio educativo. https://simon.minedu.gob.pe/

Minedu (2016). Currículo nacional. http://www.minedu.gob.pe/curriculo/pdf/programa-curriculareducacion-primaria.pdf.

Miranda, C., \& Castillo, P. (2018). Estudio metodológico de las variables que componen el constructo de "apoyo y participación de la familia en los procesos educativos". Estudios pedagógicos (Valdivia), 44(1), 115-133. http://dx.doi.org/10.4067/S0718-07052018000100115

Ormrod, J. E., Sanz, A. J. E., Soria, M. O., \& Carnicero, J. A. C. (2005). Aprendizaje humano. Madrid, Spain: Pearson Educación.

Oscco, R. (2015). Optimización del desempeño docente en la forma de atención semipresencial y el logro de competencias en los estudiantes del centro piloto Madre Teresa de Calcuta de educación básica alternativa de San Juan de Lurigancho, 2014. http://repositorio.une.edu.pe/handle/UNE/267

Pantoja, L. M. (2018). ¿Evaluación en competencias? Estudios pedagógicos, 38(1), 353-366.

Rychen, S.R. \& Salganik, L.H. (2003): A holistic model of competence. In D.S. Rychen \& L.H.Salganik (Eds) Key Competencies for a successful life and a well-functioning society. 
Gottingen: Hogrefe \& Huber. Chapter 2 pp.41-62. https://pubengine2.s3.eu-central 1.amazonaws.com/preview/99.110005/9781616762728_preview.pdf

Rodríguez, M. A. M. (1999). El enfoque sociocultural en el estudio del desarrollo y la educación. Redie. Revista Electrónica de Investigación Educativa, 1(1), 16-37

Saldarriaga, P. J., Bravo, G. D. R., \& Loor, M. R. (2016). La teoría constructivista de Jean Piaget y su significación para la pedagogía contemporánea. Dominio de las Ciencias, 2(3 Especial), 127-137. https://dominiodelasciencias.com/ojs/index.php/es/article/view/298.

Sánchez, P., De Andrés, C, \& Paredes, J. (2018). El papel de la familia en el desarrollo de la competencia digital. Análisis de cuatro casos. Digital Education Review, (34), 44-58. https://dialnet.unirioja.es/servlet/articulo?codigo $=6765346$

Silva Laya, M. (2016). Competencias de estudiantes iberoamericanos al finalizar la educación secundaria alta. Perfiles educativos, 38(152), 88-108. http://www.scielo.org.mx/pdf/peredu/v38n152/0185-2698-peredu-38-152-00088.pdf

Tobón, S. T., Prieto, J. H. P., \& Fraile, J. A. G. (2010). Secuencias didácticas: aprendizaje y evaluación de competencias. México: Pearson educación.

Tobón, S. T. (2015). Formación integral y competencias. Editorial Macro.

Zúñiga, A., Leiton de Sulia, R., \& Naranjo, J. A. (2014). Del sistema educativo tradicional hacia la formación por competencias: Una mirada a los procesos de enseñanza aprendizaje de las ciencias en la educación secundaria de Mendoza Argentina y San José de Costa Rica. Revista Eureka sobre Enseñanza y Divulgación de las Ciencias 11(2), 145-159. https://revistas.uca.es/index.php/eureka/article/view/2872/2531

Anexo

\begin{tabular}{|c|c|c|c|c|c|}
\hline Instrumento & rificar el logro de & npetencias e & la estrategi: & Aprendo & n Casa \\
\hline DIMENSIÓN & INDICADOR & \multicolumn{4}{|c|}{ ÍTEMS } \\
\hline \multirow{8}{*}{ Cognitiva } & \multirow[t]{3}{*}{$\begin{array}{l}\text { Desarrollo de la } \\
\text { inteligencia }\end{array}$} & \multicolumn{4}{|c|}{$\begin{array}{l}\text { Las competencias priorizadas en la estrategia } \\
\text { Aprendo en Casa apuntan al desarrollo de la } \\
\text { inteligencia humana. }\end{array}$} \\
\hline & & $\begin{array}{l}\text { Totalmente } \\
\text { en } \\
\text { desacuerdo }\end{array}$ & $\begin{array}{l}\text { En } \\
\text { desacuerdo }\end{array}$ & $\begin{array}{l}\text { De } \\
\text { acuerdo }\end{array}$ & $\begin{array}{l}\text { Totalmente } \\
\text { de acuerdo }\end{array}$ \\
\hline & & 1 & 2 & 3 & 4 \\
\hline & \multirow{3}{*}{$\begin{array}{l}\text { Habilidades de } \\
\text { pensamiento }\end{array}$} & \multicolumn{4}{|c|}{$\begin{array}{l}\text { La retroalimentación realizada por los docentes } \\
\text { promueve el pensamiento crítico-reflexivo. }\end{array}$} \\
\hline & & $\begin{array}{l}\text { Totalmente } \\
\text { en } \\
\text { desacuerdo }\end{array}$ & $\begin{array}{l}\text { En } \\
\text { desacuerdo }\end{array}$ & $\begin{array}{l}\text { De } \\
\text { acuerdo }\end{array}$ & $\begin{array}{l}\text { Totalmente } \\
\text { de acuerdo }\end{array}$ \\
\hline & & 1 & 2 & 3 & 4 \\
\hline & $\begin{array}{l}\text { Estrategias } \\
\text { cognitivas }\end{array}$ & \multicolumn{4}{|c|}{$\begin{array}{l}\text { Las estrategias cognitivas aplicadas por el docente } \\
\text { permiten el logro de competencias a través de } \\
\text { Aprendo en Casa. }\end{array}$} \\
\hline & & $\begin{array}{l}\text { Totalmente } \\
\text { en } \\
\text { desacuerdo }\end{array}$ & $\begin{array}{l}\text { En } \\
\text { desacuerdo }\end{array}$ & $\begin{array}{l}\text { De } \\
\text { acuerdo }\end{array}$ & $\begin{array}{l}\text { Totalmente } \\
\text { de acuerdo }\end{array}$ \\
\hline
\end{tabular}




\begin{tabular}{|c|c|c|c|c|c|}
\hline & & 1 & 2 & 3 & 4 \\
\hline \multirow{9}{*}{ Comunicativa } & \multirow{3}{*}{$\begin{array}{l}\text { Producción de } \\
\text { textos }\end{array}$} & \multicolumn{4}{|c|}{$\begin{array}{l}\text { La producción de textos permite el logro de } \\
\text { competencias comunicativas en Aprendo en Casa. }\end{array}$} \\
\hline & & $\begin{array}{l}\text { Totalmente } \\
\text { en } \\
\text { desacuerdo }\end{array}$ & $\begin{array}{l}\text { En } \\
\text { desacuerdo }\end{array}$ & $\begin{array}{l}\text { De } \\
\text { acuerdo }\end{array}$ & $\begin{array}{l}\text { Totalmente } \\
\text { de acuerdo }\end{array}$ \\
\hline & & 1 & 2 & 3 & 4 \\
\hline & \multirow{3}{*}{ Comprensión } & \multicolumn{4}{|c|}{$\begin{array}{l}\text { La retroalimentación realizada por los docentes } \\
\text { enfatiza en la comprensión de textos escritos. }\end{array}$} \\
\hline & & $\begin{array}{l}\text { Totalmente } \\
\text { en } \\
\text { desacuerdo }\end{array}$ & $\begin{array}{l}\text { En } \\
\text { desacuerdo }\end{array}$ & $\begin{array}{l}\text { De } \\
\text { acuerdo }\end{array}$ & $\begin{array}{l}\text { Totalmente } \\
\text { de acuerdo }\end{array}$ \\
\hline & & 1 & 2 & 3 & 4 \\
\hline & \multirow[t]{3}{*}{ Análisis } & \multicolumn{4}{|c|}{$\begin{array}{l}\text { Las evidencias recogidas en el portafolio de los } \\
\text { estudiantes generan el desarrollo de la capacidad } \\
\text { de análisis de los estudiantes. }\end{array}$} \\
\hline & & $\begin{array}{l}\text { Totalmente } \\
\text { en } \\
\text { desacuerdo }\end{array}$ & $\begin{array}{l}\text { En } \\
\text { desacuerdo }\end{array}$ & $\begin{array}{l}\text { De } \\
\text { acuerdo }\end{array}$ & $\begin{array}{l}\text { Totalmente } \\
\text { de acuerdo }\end{array}$ \\
\hline & & 1 & 2 & 3 & 4 \\
\hline \multirow[t]{9}{*}{$\begin{array}{c}\text { Social. } \\
\checkmark\end{array}$} & \multirow[t]{3}{*}{$\begin{array}{l}\checkmark \quad \text { Costumbres } \\
\text { sociales }\end{array}$} & \multicolumn{4}{|c|}{$\begin{array}{l}\text { Las interacciones que se dan en las diversas } \\
\text { familias permiten el logro de competencias de } \\
\text { Aprendo en Casa. }\end{array}$} \\
\hline & & $\begin{array}{l}\text { Totalmente } \\
\text { en } \\
\text { desacuerdo }\end{array}$ & $\begin{array}{l}\text { En } \\
\text { desacuerdo }\end{array}$ & $\begin{array}{l}\text { De } \\
\text { acuerdo }\end{array}$ & $\begin{array}{l}\text { Totalmente } \\
\text { de acuerdo }\end{array}$ \\
\hline & & 1 & 2 & 3 & 4 \\
\hline & \multirow[t]{3}{*}{$\begin{array}{l}\text { Diferencias } \\
\text { culturales }\end{array}$} & \multicolumn{4}{|c|}{$\begin{array}{l}\text { Las diferencias culturales que presentan las } \\
\text { familias son un impedimento para el logro de } \\
\text { competencias en la estrategia Aprendo en Casa. }\end{array}$} \\
\hline & & $\begin{array}{l}\text { Totalmente } \\
\text { en } \\
\text { desacuerdo }\end{array}$ & $\begin{array}{l}\text { En } \\
\text { desacuerdo }\end{array}$ & $\begin{array}{l}\text { De } \\
\text { acuerdo }\end{array}$ & $\begin{array}{l}\text { Totalmente } \\
\text { de acuerdo }\end{array}$ \\
\hline & & 1 & 2 & 3 & 4 \\
\hline & \multirow[t]{3}{*}{$\begin{array}{ll}\checkmark & \text { Interacción }\end{array}$} & \multicolumn{4}{|c|}{$\begin{array}{l}\text { La ausencia de los padres de familia en el apoyo a } \\
\text { la estrategia Aprendo en Casa no permite el logro } \\
\text { de competencias. }\end{array}$} \\
\hline & & $\begin{array}{l}\text { Totalmente } \\
\text { en } \\
\text { desacuerdo }\end{array}$ & $\begin{array}{l}\text { En } \\
\text { desacuerdo }\end{array}$ & $\begin{array}{l}\text { De } \\
\text { acuerdo }\end{array}$ & $\begin{array}{l}\text { Totalmente } \\
\text { de acuerdo }\end{array}$ \\
\hline & & 1 & 2 & 3 & 4 \\
\hline \multirow{3}{*}{ Ética } & \multirow{3}{*}{$\begin{array}{l}\text { Construcción de } \\
\text { la norma }\end{array}$} & \multicolumn{4}{|c|}{$\begin{array}{l}\text { La Estrategia Aprendo en Casa permite construir } \\
\text { normas de conducta para facilitar el logro de } \\
\text { competencias en las sesiones planificadas. }\end{array}$} \\
\hline & & $\begin{array}{l}\text { Totalmente } \\
\text { en } \\
\text { desacuerdo }\end{array}$ & $\begin{array}{l}\text { En } \\
\text { desacuerdo }\end{array}$ & $\begin{array}{l}\text { De } \\
\text { acuerdo }\end{array}$ & $\begin{array}{l}\text { Totalmente } \\
\text { de acuerdo }\end{array}$ \\
\hline & & 1 & 2 & 3 & 4 \\
\hline
\end{tabular}




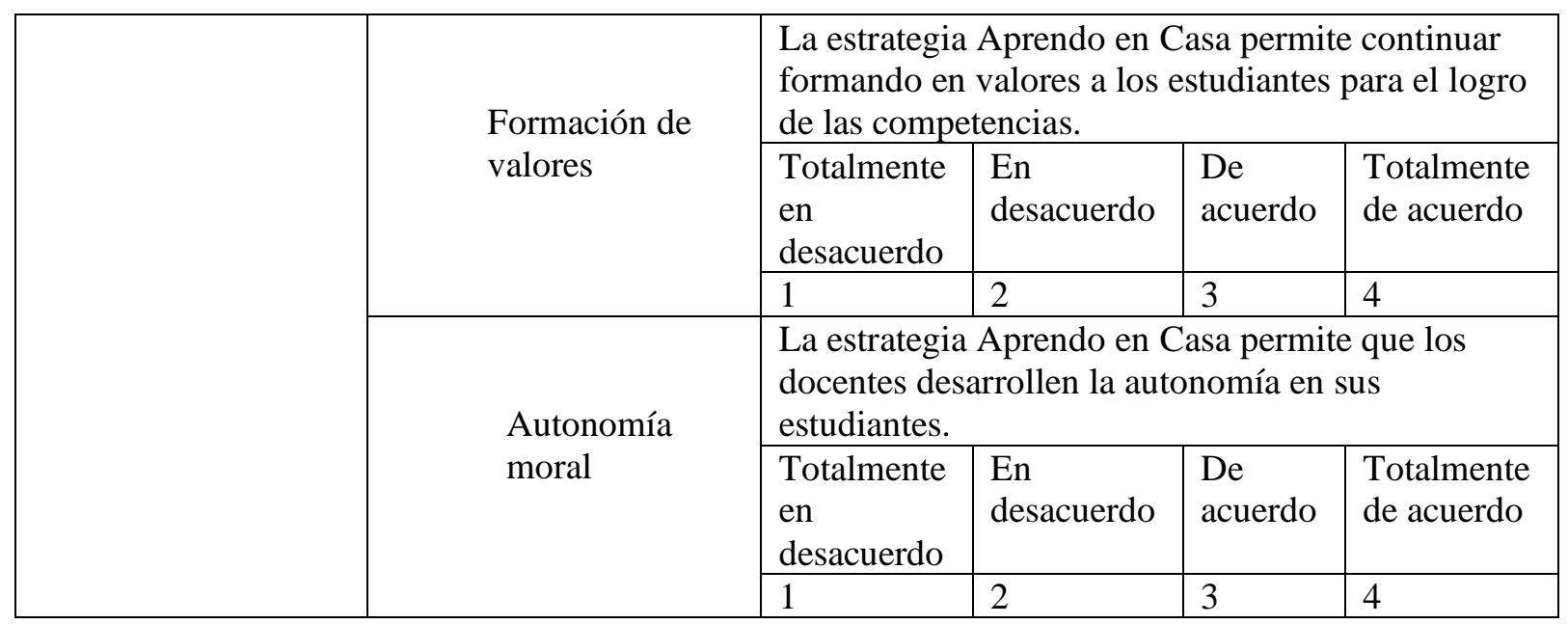

